Nainaru Tarakaramu, P.V. Satya Narayana*, and Bhumarapu Venkateswarlu

\title{
Numerical simulation of variable thermal conductivity on 3D flow of nanofluid over a stretching sheet
}

https://doi.org/10.1515/nleng-2020-0011

Received Sep 21, 2019; accepted Mar 11, 2020.

\begin{abstract}
The present investigation deals with the steady three-dimensional flow and heat transfer of nanofluids due to stretching sheet in the presence of magnetic field and heat source. Three types of water based nanoparticles namely, copper $(\mathrm{Cu})$, aluminium oxide $\left(\mathrm{Al}_{2} \mathrm{O}_{3}\right)$, and titanium dioxide $\left(\mathrm{TiO}_{2}\right)$ are considered in this study. The temperature dependent variable thermal conductivity and thermal radiation has been introduced in the energy equation. Using suitable similarity transformations the dimensional non-linear expressions are converted into dimensionless system and are then solved numerically by RungeKutta-Fehlberg scheme along with well-known shooting technique. The impact of various flow parameters on axial and transverse velocities, temperature, surface frictional coefficients and rate of heat transfer coefficients are visualized both in qualitative and quantitative manners in the vicinity of stretching sheet. The results reviled that the temperature and velocity of the fluid rise with increasing values of variable thermal conductivity parameter. Also, the temperature and normal velocity of the fluid in case of $\mathrm{Cu}$-water nanoparticles is more than that of $\mathrm{Al}_{2} \mathrm{O}_{3}$ - water nanofluid. On the other hand, the axial velocity of the fluid in case of $\mathrm{Al}_{2} \mathrm{O}_{3}$ - water nanofluid is more than that of $\mathrm{TiO}_{2}$ nanoparticles. In addition, the current outcomes are matched with the previously published consequences and initiate to be a good contract as a limiting sense.
\end{abstract}

Keywords: 3D flow; MHD; nanofluid; variable thermal conductivity; radiation; heat source

Nainaru Tarakaramu, Department of Mathematics, SAS, VIT University, Vellore-632 014, T.N, India

*Corresponding Author: P.V. Satya Narayana, Department of Mathematics, SAS, VIT University, Vellore-632 014, T.N, India, E-mail: pvsatya8@yahoo.co.in, Tel.: +919789574488

Bhumarapu Venkateswarlu, Department of Mathematics, Walchand Institute of Technology, Solapur-413006, M.H, India

\section{Introduction}

Recently, the study of nanofluid flows generated due to a stretching sheet has been attracted by the many researchers because of their fascinating engineering and industrial applications such as microelectronics, engine cooling, refrigerator and fuel cells. The fundamental point of using nanofluids in such frameworks are to upgrade the thermal conductivity and enhancing the heat transfer capacity in order to accomplish better cooling. The reviews [1-4] reviled that the heat exchange performance of the nanoparticles (like $\mathrm{Cu}, \mathrm{Al}_{2} \mathrm{O}_{3}, \mathrm{TiO}_{2}$ ) are more in comparison with the base fluids (such as water, glycol, toluene and oil etc.). Rehman et al. [5] examined the effect of thermal radiation on a stretching sheet in the presence of nanofluid. They originate that the $C$ unanoparticles enhancing heat transfer rate with minimal entropy generation. Recently, many researchers [6-10] have paid their interest on nanofluid flows with different boundary conditions on different models. Melting effect on heat transfer flow of nanofluid with Buongiorno model was studied by Sheikholeslami et al. [11]. Umavathi et al. [12] considered the convective heat transfer flow of nanofluid by using Darcy-Forchheimer-Brinkman model. They concluded that the flow and heat transfer characteristics enhance with increase of Darcy or Grashof or Brinkman numbers while it reduce with the inertial or viscosity ratio parameters. Iqbal et al. [13] presented the effects of heat convection on nanofluid flow over a moving Riga plate. They solved the governing equations numerically by using finite difference scheme Keller Box method. Ahmad Khan et al. [14] employed two different models for 3D rotating flow of effective thermal conductivity of nanofluids. FalknerSkan flow of MHD Carreau nanofluid was investigated by Masood Khan et al. [15]. They applied two numerical techniques namely shooting and Newton's Raphson methods in their study. Hayat et al. [16] developed the 3D rotating flow of Maxwell fluid through submersion nanoparticle. Impact of MHD on heat transfer flow of a nanofluid generated by a stretching sheet was studied by [17-19]. 
The influence of temperature dependent thermal conductivity in the presence of nanofluids is most important because it change with temperature. The CattaneoChristov heat flux theory with Jeffery liquid was reported by Meraj et al. [20]. They initiate the thermal boundary layer thickness is high for heat conduction of Fourier's law with comparison of Cattaneo-Christov heat flux model. But the concentration plays an opposite behaviour with reaction parameters. Hayat et al. [21] examined the CattaneoChristov heat flux on Jeffrey fluid flow with variable thermal conductivity. Ramzan et al. [22] analyzed the effects of temperature dependent thermal conductivity on nonNewtonian nanofluid flow past a moving surface. They found that the temperature increases with the rise of thermal radiation. Srinivas Reddy et al. [23] examined the heat transfer characteristics of Williamson nanofluid over a stretching sheet. They solved the equations by spectral quasi-linearization method by considering the variable thermal conductivity. Dyugaev et al. [24] studied the influence of thermal conductivity and viscosity of fluid with ultra - fine particles. Some of the scientist's worked on the influence of thermal radiation on free convection flow through different models [25-29].

Animasaun [30] explored the influence of chemical reaction and thermophoresis on the nanofluid boundary layer flow over an upper horizontal surface of paraboloid of revolution. They solved the buoyancy model by using R-K-4-S-T (Runge-Kutta fourth order along with shooting technique) with volume fraction $(\phi)$ defined as $0 \% \leq \phi \leq$ $.8 \%$. Three kinds of water-based nanofluids $\left(\mathrm{Al}_{2} \mathrm{O}_{3}, \mathrm{Cu}\right.$, and $\mathrm{TiO}_{2}$ nanoparticles) with volume fraction $10 \%$ and $20 \%$ in a cubical enclosure has been analysed by Boutra et al. [31]. They implemented a boundary element method to simulate the flow and estimate the thermal conductivity and viscosity of nanofluids. The natural convection of $\mathrm{Al}_{2} \mathrm{O}_{3}$ /water nanofluids with volume fractions up to 3\% in a cubic cavity was proposed by Saghir et al. [32]. Purusothaman et al. [33] presented the natural convection of nanofluids in a cavity model by finite volume method. Later, Snoussi et al. [34], Sheikholeslami and Ellahi [35] and Kolsi et al. [36-38] established convection flow of $\mathrm{Al}_{2} \mathrm{O}_{3}$ /water nanofluids in a cube cavity with a maximum volume fraction up to $20 \%$. Meng and Li [39] simulated the free convection of $\mathrm{Al}_{2} \mathrm{O}_{3}$ /water nanofluids with two volume concentrations of 1 and $4 \%$ in a horizontal cylinder. Ho et al. [40] developed the correlations for thermal conductivity and viscosity.

The literature reviles that the thermal conductivity of nanofluids are not constant and it varies linearly with the temperature and they play an important role in many engineering and industrial applications. Hence, the main ob- jective of current analysis is fill the gap in the literature by studying the influence of variable thermal conductivity on three dimensional flow of MHD nanofluid (with different nanoparticles) caused by a stretching sheet with thermal radiation. Similarity transformations are applied to nonlinear partial differential equations and the transformed system can be solved numerically by Runge-KuttaFehlberg scheme with shooting technique. Expressions for various values of parameters on the velocity and temperature as well as the Nusselt number are discussed graphically.

\section{Mathematical formulation}

The steady three-dimensional flow of an electrical conducting magnetohydrodynamic nanoliquid through a stretching surface is considered in this model. The variable thermal conductivity and heat transfer of nanofluids have been studied in view of free convection. Choose a cartesian coordinates system $(x, y, z)$ in which $x$ - and $y$-axes are taken along the sheet surface in the direction of the fluid flow and $z$-axis is perpendicular to it. The stretching sheet is coincident at $z=0$ and flow dwell in the region $z>0$ as shown in Figure 1. $u_{w}^{\star}(x)=a^{\star} x$ and $v_{w}^{\star}(y)=b^{\star} y$ are stretching velocities along the fluid flow direction. Furthermore, $w^{\star}=-W^{\star}$ is mass flux velocity, considered on the sheet. Where $w^{\star}>0$ is the suction and $w^{\star}<0$ is the injection. A uniform magnetic field of strength $B_{0}$ is assumed in the direction of $z$ and normal to the surface (i.e. $x y$-plane). The induced Lorentz force and impressed electric field are neglected due to the small magnetic Reynolds number.

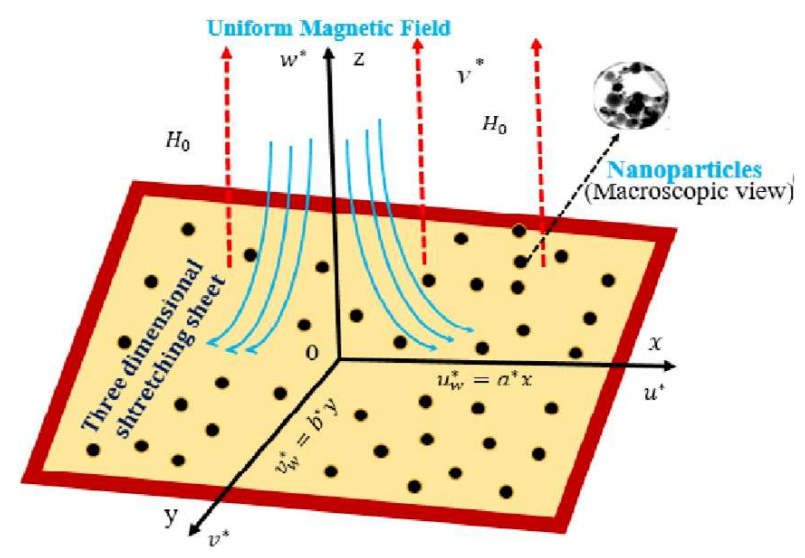

Figure 1: Physical model of the problem 
The dynamical equations of the three dimensional nanofluid flow along with heat transfer can be expressed (see Ref. [41, 42]) as

$$
\begin{gathered}
\frac{\partial u^{\star}}{\partial x}+\frac{\partial v^{\star}}{\partial y}+\frac{\partial w^{\star}}{\partial z}=0 \\
u^{\star} \frac{\partial u^{\star}}{\partial x}+v^{\star} \frac{\partial u^{\star}}{\partial y}+w^{\star} \frac{\partial u^{\star}}{\partial z}=\frac{\mu^{\star}{ }_{n f}}{\rho^{\star}} \frac{\partial^{2} u^{\star}}{\partial z^{2}} \\
+\frac{g^{\star}\left(\rho^{\star} \beta\right)_{n f}}{\rho^{\star}{ }_{n f}}\left(T^{\star}-T^{\star}{ }_{\infty}\right)-\frac{\sigma^{\star} H_{0}^{2}}{\rho^{\star}{ }_{n f}} u^{\star} \\
u^{\star} \frac{\partial v^{\star}}{\partial x}+v^{\star} \frac{\partial v^{\star}}{\partial y}+w^{\star} \frac{\partial v^{\star}}{\partial z}=\frac{\mu_{n f}^{\star}}{\rho_{n f}^{\star}} \frac{\partial^{2} v^{\star}}{\partial z^{2}} \\
+\frac{g^{\star}\left(\rho^{\star} \beta\right)_{n f}}{\rho^{\star}{ }_{n f}}\left(T^{\star}-T^{\star}{ }_{\infty}\right)-\frac{\sigma^{\star} H_{0}^{2}}{\rho^{\star}{ }_{n f}} v^{\star}
\end{gathered}
$$

$u^{\star} \frac{\partial T^{\star}}{\partial x}+v^{\star} \frac{\partial T^{\star}}{\partial y}+w^{\star} \frac{\partial T^{\star}}{\partial z}=\frac{K^{\star}{ }_{n f}}{\left(\rho^{\star} C_{p}\right)_{n f}} \frac{\partial}{\partial z}\left(\varsigma^{\star}\left(T^{\star}\right) \frac{\partial T^{\star}}{\partial z}\right)$

$$
+\frac{1}{\left(\rho^{\star} c_{p}\right)_{n f}} \frac{\partial q_{r}^{\star}}{\partial z}-\frac{Q_{0}^{\star}\left(T^{\star}-T^{\star} \infty\right)}{\left(\rho^{\star} c_{p}\right)_{n f}}
$$

The boundary conditions are defined as

$$
\left.\begin{array}{l}
u^{\star}=u_{w}^{\star}(x)=a^{\star} x \quad v^{\star}=v_{w}^{\star}(y)=b^{\star} y \\
w^{\star}=-W^{\star}, T^{\star}=T^{\star}{ }_{w} \quad \text { at } z=0 \\
u^{\star}(z) \rightarrow 0 \quad v^{\star}(z) \rightarrow 0, \quad T^{\star}(z) \rightarrow T^{\star}{ }_{\infty} \text { as } z \rightarrow \infty
\end{array}\right\}
$$

The temperature dependent thermal conductivity $\varsigma^{\star}(T)$ is defined (Ref. [41]) as

$$
\varsigma^{\star}(T)=\omega_{\infty}\left\{1+\frac{\varepsilon^{\star}}{\Delta T^{\star}}\left(T^{\star}-T_{\infty}^{\star}\right)\right\}
$$

Where $\Delta T^{\star}=T^{\star}{ }_{w}-T^{\star}{ }_{\infty}$ and $T_{w}^{\star}$ is the sheet temperature, $\omega_{\infty}$ is the conductivity of the fluid far away from the sheet.

By Rosseland approximation, the radiative heat flux $q_{r}^{\star}$ (see Ref. $\left.[43,44]\right)$ is given by

$$
\begin{aligned}
& q_{r}^{\star}=-\frac{4 \sigma^{\star}}{3 k^{\star}} \frac{\partial\left(T^{\star}\right)^{4}}{\partial z}, q_{r}^{\star}=-\frac{16 \sigma^{\star}\left(T^{\star}\right)_{\infty}^{3}}{3 k^{\star}} \frac{\partial T^{\star}}{\partial z}, \\
& \Rightarrow \frac{\partial q_{r}^{\star}}{\partial z}=-\frac{16 \sigma^{\star}\left(T^{\star}\right)_{\infty}^{3}}{3 k^{\star}} \frac{\partial^{2} T^{\star}}{\partial z^{2}}
\end{aligned}
$$

Substituting equation (7) in the energy equation (4) and it becomes

$$
\begin{aligned}
& u^{\star} \frac{\partial T^{\star}}{\partial x}+v^{\star} \frac{\partial T^{\star}}{\partial y}+w^{\star} \frac{\partial T^{\star}}{\partial z}=\frac{k^{\star}{ }_{n f}}{\left(\rho^{\star} c_{p}\right)_{n f}} \frac{\partial T^{\star}}{\partial z} \frac{\partial \varsigma^{\star}\left(T^{\star}\right)}{\partial z} \\
& +\frac{k^{\star}{ }_{n f} \varsigma^{\star}(T)}{\left(\rho^{\star} c_{p}\right)_{n f}} \frac{\partial^{2} T^{\star}}{\partial z^{2}}-\frac{Q_{0}^{\star}\left(T^{\star}-T^{\star} \infty\right)}{\left(\rho^{\star} c_{p}\right)_{n f}}-\frac{16 \sigma^{\star}\left(T^{\star}\right)_{\infty}^{3}}{3 k^{\star}\left(\rho^{\star} c_{p}\right)_{n f}} \frac{\partial^{2} T^{\star}}{\partial z^{2}}
\end{aligned}
$$

Where

$$
\left.\begin{array}{l}
\rho^{\star}{ }_{n f}=(1-\phi) \rho^{\star}{ }_{f}+\phi \rho^{\star}{ }_{s} \\
\left(\rho^{\star} c_{p}\right)_{n f}=(1-\phi)\left(\rho^{\star} c_{p}\right)_{f}+\phi\left(\rho^{\star} c_{p}\right)_{s} \\
\left(\beta^{\star}\right)_{n f}=(1-\phi)\left(\beta^{\star}\right)_{f}+\phi\left(\beta^{\star}\right)_{s} \\
\left(\rho^{\star} \beta^{\star}\right)_{n f}=(1-\phi)\left(\rho^{\star} \beta^{\star}\right)_{f}+\phi\left(\rho^{\star} \beta^{\star}\right)_{s}, \\
\frac{k^{\star}{ }_{n f}}{k^{\star}}=\frac{k^{\star}{ }_{s}+2 k^{\star}}{k_{f}-2 \phi\left(k^{\star}+2 k^{\star}-k^{\star}{ }_{s}+2 \phi\left(k^{\star}{ }_{f}-k^{\star}{ }_{s}\right)\right.}
\end{array}\right\}
$$

The effective dynamic viscosity of the nanofluid is described as [see Ref. [5, 6, 41])

$$
\mu^{\star}{ }_{n f}=\mu_{f}^{\star}\left(1+39.11 \phi+533.9 \phi^{2}\right)
$$

The similarity variables are

$$
\left.\begin{array}{ll}
u^{\star}=a^{\star} x f^{\prime}(\eta) \quad v^{\star}=a^{\star} y g^{\prime}(\eta) & \theta(\eta)=\frac{T^{\star}-T_{\infty}^{\star}}{T^{\star} w^{-T_{\infty}}} \\
w^{\star}=-\sqrt{a^{\star} v^{\star}}(f(\eta)+g(\eta)) & \eta=\sqrt{\frac{a^{\star}}{v^{*}} z}
\end{array}\right\}
$$

With the help of the above relations, the governing equations finally reduce to

$$
f^{\prime \prime \prime}+\varepsilon_{4}\left\{\varepsilon_{1}\left\{(f+g) f^{\prime \prime}-\left(f^{\prime}\right)^{2}\right\}+\varepsilon_{2} y \theta-M f^{\prime}\right\}=0
$$

$$
\begin{gathered}
g^{\prime \prime \prime}+\varepsilon_{4}\left\{\varepsilon_{1}\left\{(f+g) g^{\prime \prime}-\left(g^{\prime}\right)^{2}\right\}+\varepsilon_{2} y \theta-M g^{\prime}\right\}=0 \\
\left\{A R_{d}\left(1+\varepsilon^{\star} \theta\right)-\frac{4}{3}\right\} \theta^{\prime \prime}+\varepsilon_{3} \operatorname{Pr}_{d}(f+g) \theta^{\prime} \\
+\varepsilon^{*} A R_{d}\left(\theta^{\prime}\right)^{2}-H \operatorname{PrR}_{d} \theta=0
\end{gathered}
$$

The corresponding boundary conditions can be written as

$$
\left.\begin{array}{c}
f^{\prime}(\eta)=1 \quad g^{\prime}(\eta)=\lambda \quad f(\eta)+g(\eta)=S \quad \theta(\eta)=1 \\
\text { at } \eta=0 \\
f^{\prime}(\eta) \rightarrow 0 \quad g^{\prime}(\eta) \rightarrow 0 \quad \theta(\eta) \rightarrow 0 \quad \text { as } \eta \rightarrow \infty
\end{array}\right\}
$$

Where $\lambda$ is the stretching ratio parameter, $S$ is a constant mass wall transfer with $S>0$ for suction, $S<0$ for injunction and impermeable plate $S=0$ and

$$
A=\frac{k_{n f}^{\star}}{k_{f}^{\star}} R_{d}=\frac{k^{\star} k_{\infty}^{\star} k_{f}^{\star}}{4 \sigma^{\star} T_{\infty}^{\star 3}} M=\frac{\sigma^{\star} B_{0}^{2}}{a^{\star} \rho_{f}^{\star}} H=\frac{Q_{0}^{\star}}{a^{\star}\left(\rho^{\star} c_{p}\right)_{f}}
$$

$$
\lambda=\frac{b^{\star}}{a^{\star}} \operatorname{Pr}=\frac{v_{f}^{\star}\left(\rho^{\star} c_{p}\right)_{f}}{k_{\infty}^{\star} k^{\star}{ }_{f}} S=\frac{W^{\star}}{\sqrt{a^{\star} v_{f}^{\star}}} y=\frac{g^{\star} \beta_{f}^{\star}\left(T^{\star}-T^{\star}{ }_{\infty}\right)}{\rho_{f} a^{\star} u^{\star}}
$$

$\varepsilon_{1}=1-\phi+\left(\rho^{\star}{ }_{s} / \rho_{f}^{\star}\right) \phi, \quad \varepsilon_{3}=1-\phi+\left\{\left(\rho^{\star} c_{p}\right)_{s} /\left(\rho^{\star} c_{p}\right)_{f}\right\} \phi$

$\varepsilon_{2}=1-\phi+\left\{\left(\rho^{\star} \beta^{\star}\right)_{s} /\left(\rho^{\star} \beta^{\star}\right)_{f}\right\} \phi, \quad \varepsilon_{4}=\frac{1}{1+39.11 \phi+533.9 \phi^{2}}$ 
The surface frictional coefficients $C_{f x}, C_{f y}$ and rate of heat transfer $N u_{x}$ are respectively, defined as follows

$$
C_{f x}=\frac{\tau_{w x}}{\rho^{\star}{ }_{f}\left(U_{w}^{\star}\right)^{2}}, C_{f y}=\frac{\tau_{w y}}{\rho^{\star}{ }_{f}\left(V_{w}^{\star}\right)^{2}}, N u_{x}=\frac{x q_{w}}{k_{f}^{\star}\left(T^{\star}{ }_{w}-T^{\star} \infty\right)}
$$

Where $\tau_{w x}=\mu^{\star}{ }_{n f}\left\{\frac{\partial u_{*}}{\partial z}\right\}_{z=0}$ and $\tau_{w y}=\mu^{\star}{ }_{n f}\left\{\frac{\partial v_{*}}{\partial z}\right\}_{z=0}$ are the wall shear stresses along $x$ and $y$-axis of the stretching surface and $q_{w}=-k_{n f}^{\star}\left(1+R_{d}\right)\left\{\frac{\partial T^{\star}}{\partial z}\right\}_{z=0}$ is the wall flux from the stretching surface.

The non-dimensional form of surface friction coefficients and heat transfer coefficients are defined as

$$
\left.\begin{array}{l}
\sqrt{\operatorname{Re}_{x}} C_{f x}=\left(1+39.11 \phi+533.9 \phi^{2}\right) f^{\prime \prime}(0), \\
\sqrt{\operatorname{Re}_{y}} C_{f y}=\frac{1}{\lambda^{3 / 2}}\left(1+39.11 \phi+533.9 \phi^{2}\right) g^{\prime \prime}(0) \\
\sqrt{\operatorname{Re}_{y}} N u_{x}=-A(1+R) \theta^{\prime}(0)
\end{array}\right\}
$$

Where $\operatorname{Re}_{x}=\frac{U^{*}{ }^{x} X}{v_{f}^{*}}$ and $\operatorname{Re}_{y}=\frac{V^{*}{ }^{*} y}{v_{f}^{*}}$ are local Reynolds number.

It is observed that for $\lambda=0$, present problem reduces to the case of two dimensional linear stretching work, while $\lambda=1$, sheet is axisymmetric case where sheet is stretched in $x$ and $y$ directions with the same values and if $\lambda$ is neither zero or one then the flow behaviour along both the directions will be different.

\section{Numerical analysis}

The numerical results established to the coupled non-linear ODE's (12)-(14) along with the corresponding conditions Eq. (15) are solved using shooting procedure with the support of $4^{\text {th }}$ order Runge-Kutta-Fehlberg scheme with $[0,15]$ as the domain of the problem instead of $[0, \infty]$. The resulting ODE's and boundary conditions are reduced to the first order system by choosing $f$ by $\zeta_{1}, g$ by $\zeta_{4}, \theta$ by $\zeta_{7}$. Thus we obtain

$$
\left.\begin{array}{ll}
\zeta_{1}^{1}=\zeta_{2} & \zeta_{1}(0)=0 \\
\zeta_{2}^{1}=\zeta_{3} & \zeta_{2}(0)=1 \\
\zeta_{3}^{1}=-\varepsilon^{\star}\left\{\varepsilon_{1} \star\left(\left(\zeta_{1}+\zeta_{5}\right) \star \zeta_{3}^{\prime \prime}-\left(\zeta_{2}\right)^{2}\right)+y^{\star} \varepsilon_{2} \star \theta-M \star \zeta_{2}\right\} & \zeta_{3}(0)=\alpha_{1} \\
\zeta_{4}^{1}=\zeta_{5} & \zeta_{1}(0)+\zeta_{4}(0)=S \\
\zeta_{5}^{1}=\zeta_{6} & \zeta_{5}(0)=\lambda \\
\zeta_{6}^{1}=-\varepsilon^{\star}\left\{\varepsilon_{1} \star\left(\left(\zeta_{1}+\zeta_{5}\right)^{\star} \zeta_{6}^{\prime \prime}-\left(\zeta_{5}\right)^{2}\right)+y^{\star} \varepsilon_{2} \star \theta-M \star \zeta_{5}\right\} & \zeta_{6}(0)=\alpha_{2} \\
\zeta_{7}^{1}=\zeta_{8} & \zeta_{7}(0)=1 \\
\zeta_{8}^{1}=\left(-1 /\left(R_{d} \star A \star\left(1+\varepsilon^{\star} \theta\right)-(4 / 3)\right)\right)\left\{\begin{array}{ll}
(f+g)^{\star} \varepsilon_{3}{ }^{\star} R_{d} \star \operatorname{Pr}^{\star} \theta^{\prime} \\
+A^{\star} \varepsilon^{\star} R_{d} \star\left(\theta^{\prime}\right)^{2}-H^{\star} \operatorname{Pr} R_{d} \star \theta
\end{array}\right\} & \zeta_{8}(0)=\alpha_{3}
\end{array}\right\}
$$

Where $\alpha_{1}, \alpha_{2}$ and $\alpha_{3}$ are shooting specifications and $\left.\zeta_{2}(\infty)=0, \zeta_{5}(\infty)=0, \zeta_{7}(\infty)=0\right\}$

\section{Results and discussion}

The transformed equations (12), (13) and (14) with boundary conditions (15) have been solved numerically by RungeKutta-Fehlberg method along with shooting technique. The influence of different flow parameters on the axial and normal velocities, temperature, surface frictional coefficients and rate of heat transfer are discussed graphically in Figures 2-11. The thermophysical properties of different nanofluids are defined in Table 1 . The validity of the current work outcomes are compared with those of Magyari and Keller [45] and Liu et al. [46] in Table 2 and Table 3 respectively. It is observed that the current results are in good agreement with those existing results. 
Table 1: Thermo-physical Properties of the base fluids and nanoparticles.

\begin{tabular}{|c|c|c|c|c|c|}
\hline \multirow{2}{*}{$\begin{array}{c}\text { Physical } \\
\text { Properties }\end{array}$} & \multirow{2}{*}{$\frac{\text { Base fluid }}{\text { Water }}$} & \multicolumn{4}{|c|}{ Nanoparticles } \\
\hline & & Copper & Silver & Aluminium oxide & Titanium dioxide \\
\hline$\rho\left(\mathbf{k g} / \mathbf{m}^{3}\right)$ & 997 & 385 & 10,500 & 765 & 686.2 \\
\hline$\left.C_{p} \mathbf{(} / \mathbf{k g}-\mathbf{K}\right)$ & 4179 & 8933 & 235 & 3970 & 4250 \\
\hline$k(\mathbf{W} / \mathbf{m}-\mathbf{K})$ & 0.613 & 400 & 429 & 40 & 8.9538 \\
\hline
\end{tabular}

Table 2: Comparison of $-\theta^{\prime}(0)$ with distinct value of $\operatorname{Pr}$ for $\lambda=0$

\begin{tabular}{ccc}
\hline Pr & Magyari and Keller [45] & Present \\
\hline $\mathbf{1}$ & -0.5496 & -0.549643 \\
\hline $\mathbf{5}$ & -1.5212 & -1.521243 \\
\hline $\mathbf{1 0}$ & -2.2900 & -2.29000 \\
\hline
\end{tabular}

Table 3: Temperature gradient- $\theta^{\prime}(0)$ for distinct values of $\lambda$ when $\operatorname{Pr}=0.7$

\begin{tabular}{ccc}
\hline$\lambda$ & Liu et al. [46] & Present \\
\hline $\mathbf{0}$ & -0.4258 & -0.4258380 \\
\hline $\mathbf{1}$ & -0.6020 & -0.602000 \\
\hline
\end{tabular}

Figures 2(a)-2(c) display the influence of $M$ on both axial and transverse velocities $\left(f^{\prime}(\eta), g^{\prime}(\eta)\right)$ and temperature $(\theta(\eta))$ profiles respectively for two distinct nanoparticles, namely $\mathrm{Cu}$ and $\mathrm{TiO}_{2}$. It is noticed that the velocity profiles declines in both the directions and temperature increases by increasing values of $M$. This is because, $M$ is inversely proportional to the density by $M=\frac{\sigma B_{0}^{2}}{a \rho_{f}}$ and hence the temperature of the fluid rises with rising values of $M$. Also noticed that the heat transfer rate of $\mathrm{Cu}$ is more than that of $\mathrm{TiO}_{2}$ nanoparticles. Therefore, the two distinct nanoparticles boundary layer is vary with magnetic field parameter M.

The variations of the velocity and temperature profiles for buoyancy parameter $y$ are presented in Figures 3(a)3(c), respectively with (i.e.Cu, $\mathrm{Al}_{2} \mathrm{O}_{3}$ and $\mathrm{TiO}_{2}$ ) three different nanoparticles. It is obvious that both the velocity profiles $f^{\prime}(\eta)$ and $g^{\prime}(\eta)$ are growing and $\theta(\eta)$ demolish with rising values of $y$. This is due to the high heat source on temperature profile.

Figures 4(a)-4(c) illustrate the influence of stretching ration parameter $\lambda$ on both velocity components and temperature distributions. The velocity increases along the normal direction and temperature, velocity along axial di-

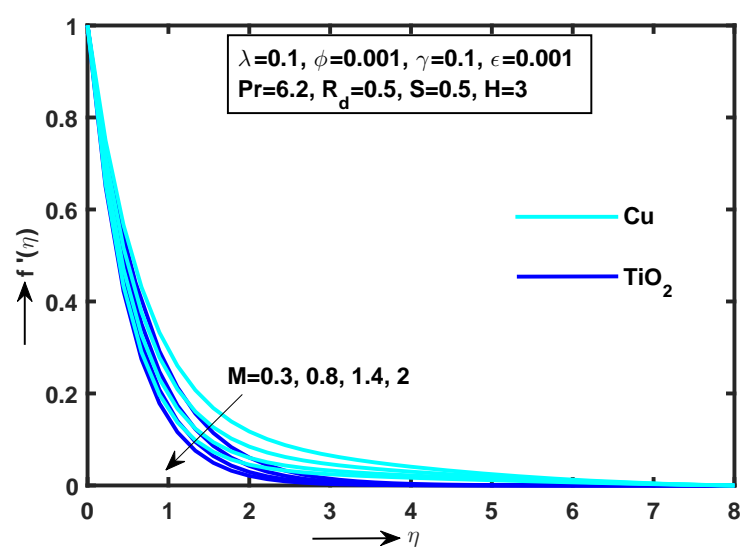

(a)

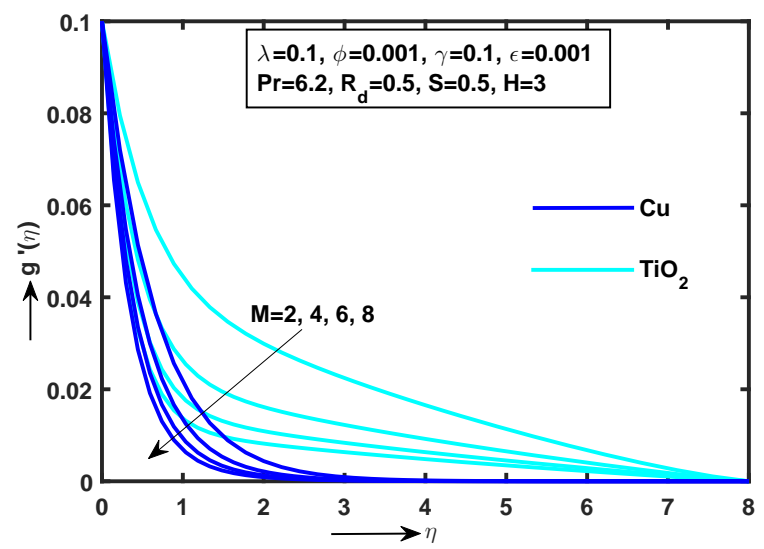

(b)

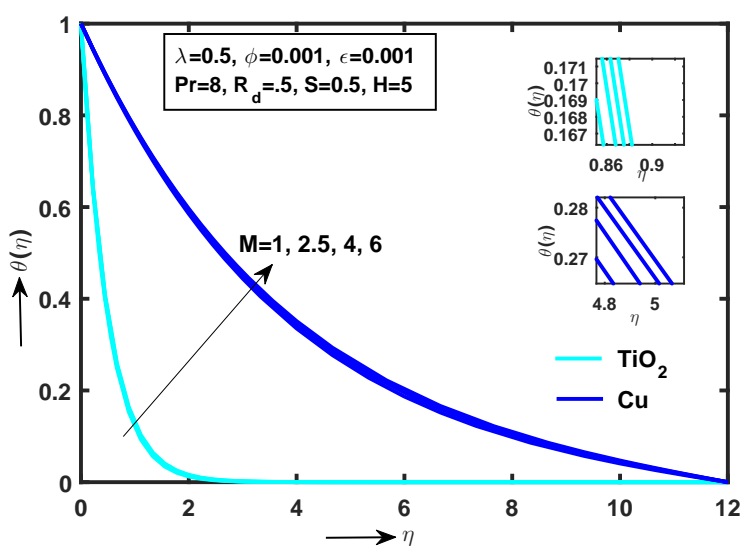

(c)

Figure 2: (a) Significance of $M$ on $f^{\prime}(\eta)$ (b) Significance of $M$ on $g^{\prime}(\eta)$ (c) Significance of $M$ on $\theta(\eta)$ 


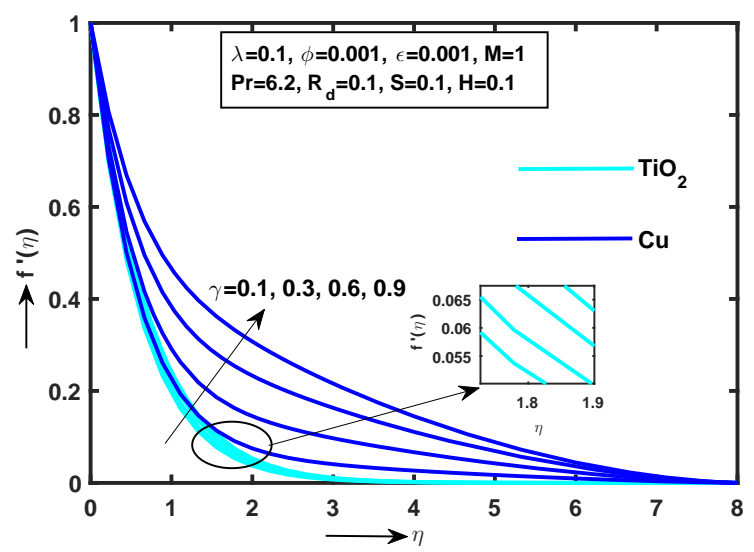

(a)

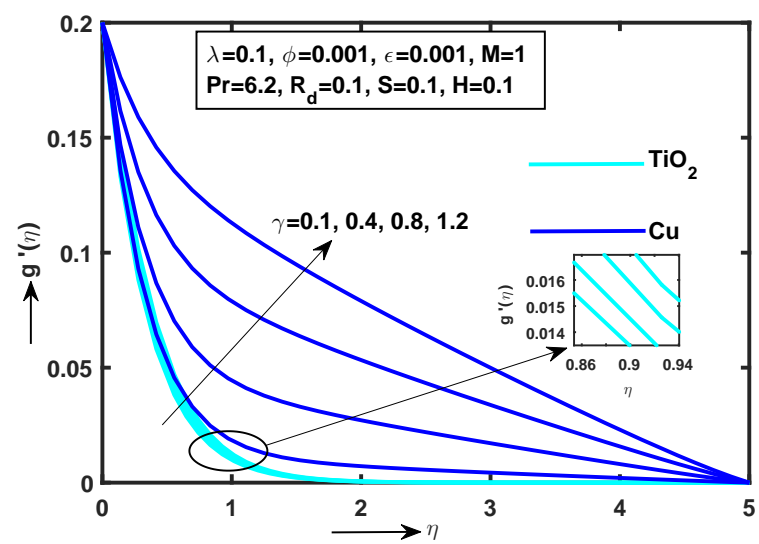

(b)

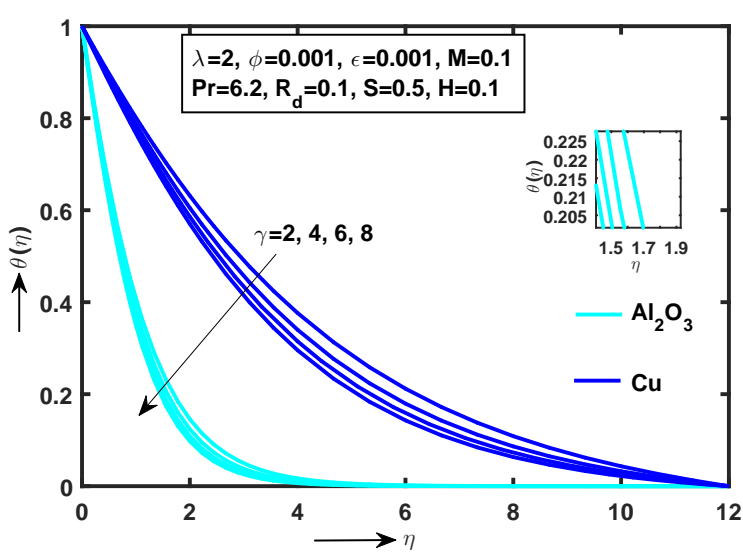

(c)

Figure 3: (a) Significance of $y$ on $f^{\prime}(\eta)$ (b) Significance of $y$ on $g^{\prime}(\eta)$ (c) Significance of $y$ on $\theta(\eta)$

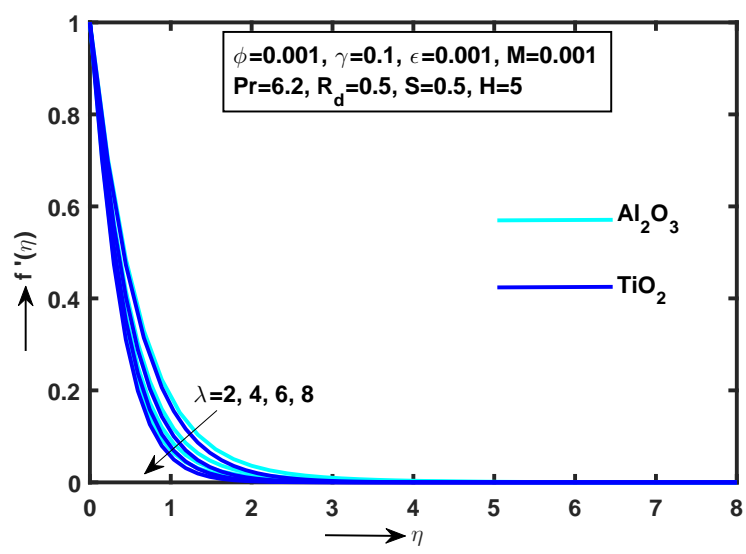

(a)

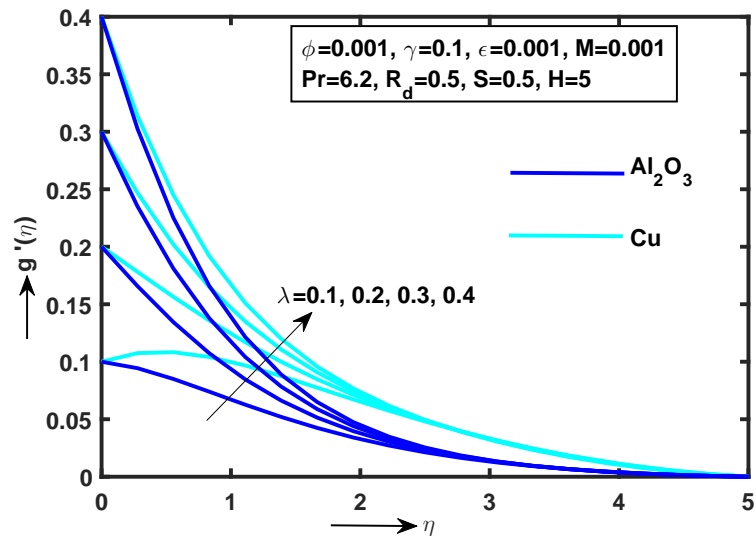

(b)

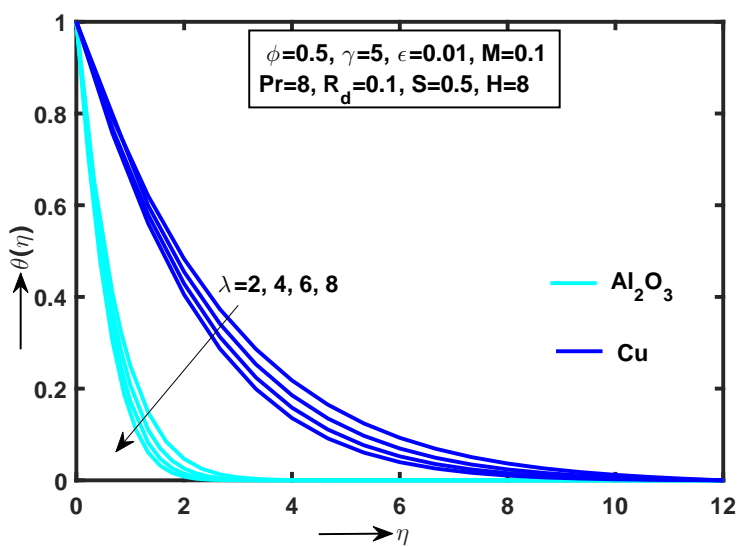

(c)

Figure 4: (a) Significance of $\lambda$ on $f^{\prime}(\eta)$ (b) Significance of $\lambda$ on $g^{\prime}(\eta)$ (c) Significance of $\lambda$ on $\theta(\eta)$ 


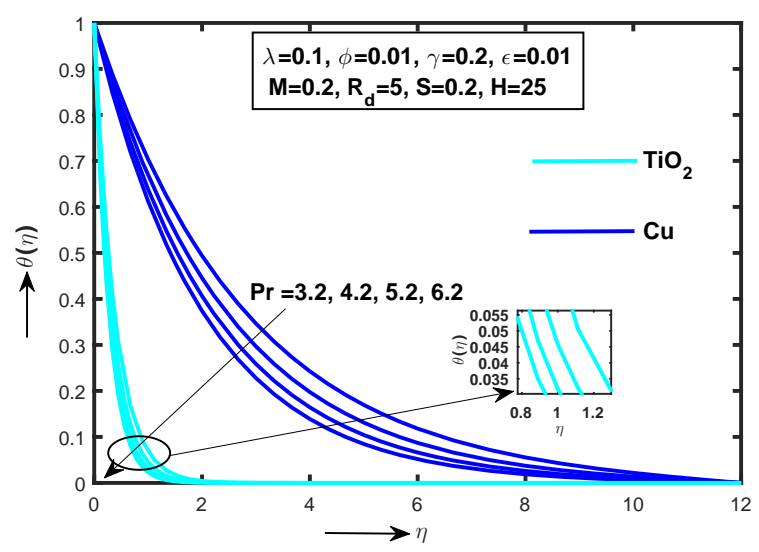

Figure 5: Significance of $\operatorname{Pr}$ on $\theta(\eta)$

rection decrease with increasing values of $\lambda$. Therefore, the Coriolis force in the fluid motion is due to stretching ratio parameter $\lambda$ which is responsible for the acceleration of the fluid motion and hence the momentum boundary layer thickness. Increasing values of stretching ratio parameter $\lambda$ causes the less heat transfer of the fluid from sheet which leads to decline in the temperature and thermal boundary layer thickness.

The variations of Pr (Prandtl number) on the velocity and temperature distributions are exhibited in Figure 5 for different $\mathrm{Cu}, \mathrm{TiO}_{2}$ nanoparticles. It is noticed that the profile $\theta(\eta)$ diminishing for ascending values of Pr. Physically, less Pr values indicate the high thermal diffusivity which causes a reduction in the fluid temperature. Hence, the thermal boundary layer thickness of Pr is proportional to the thermal diffusivity.

The nanofluid volume fraction $\phi$ characterization on both velocities and temperature profiles are elucidated respectively in Figures 6(a)-6(c). It is noticed that both the velocity profiles along axial and transverse directions increase with distinct ascending values of $\phi$ and intensifies the resistance force within the fluid and the temperature profiles has opposite behaviour. This is due to the fact that the thermal conductivity and thermal boundary layer thickness decreases with growing values of $\phi$.

Figure 7 displays the behaviour of temperature profile for different values of $\mathrm{H}$ for $\mathrm{Al}_{2} \mathrm{O}_{3}$ and $\mathrm{TiO}_{2}$ nanoparticles. The temperature of nanofluid reduces with growing values of $H$. Therefore, the heat source parameter produces the more energy to the flow and decreases the thickness of the thermal boundary layer.

Figures 8(a)-8(c) illustrate the velocity $\left(f^{\prime}(\eta), g^{\prime}(\eta)\right)$ and temperature $(\theta(\eta))$ profiles for various values of $\varepsilon$ with the nanoparticles $\mathrm{Cu}, \mathrm{Al}_{2} \mathrm{O}_{3}$ and $\mathrm{Al}_{2} \mathrm{O}_{3}, \mathrm{TiO}_{2}$ respectively. It is noticed that both the velocity profiles increases for

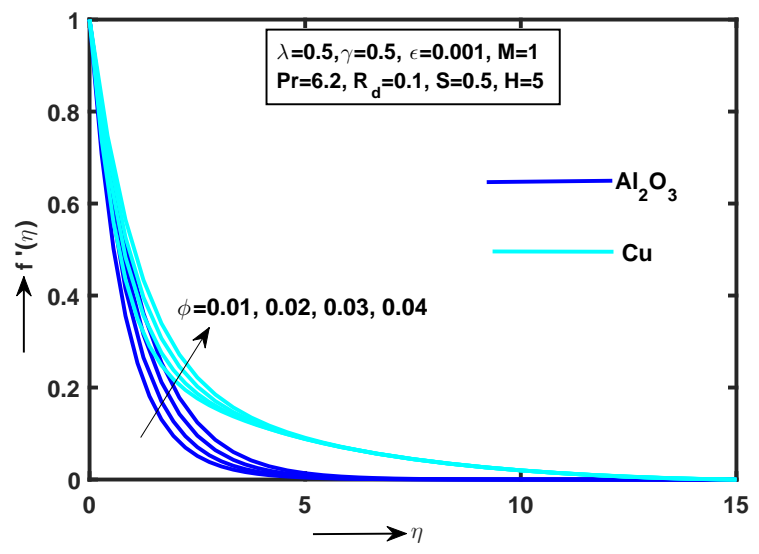

(a)

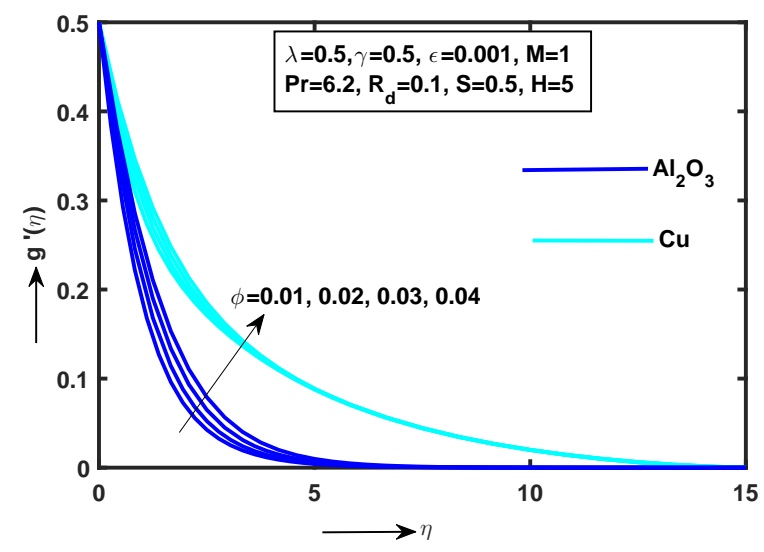

(b)

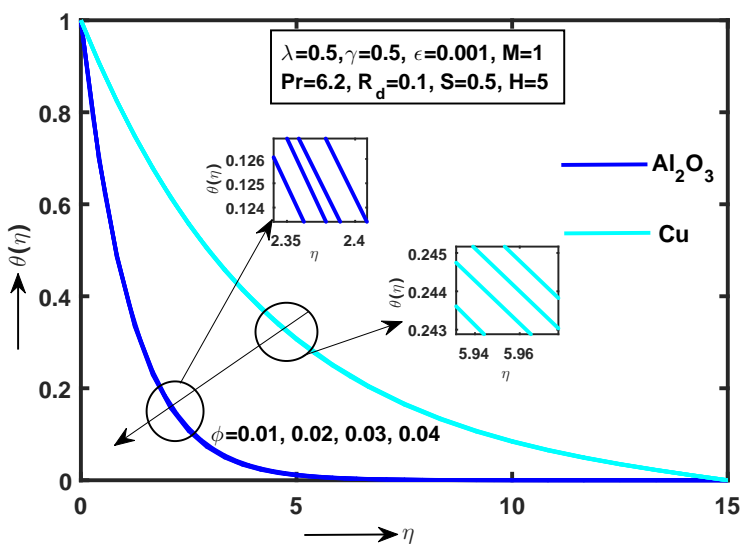

(c)

Figure 6: (a) Significance of $\phi$ on $f^{\prime}(\eta)$ (b) Significance of $\phi$ on $g^{\prime}(\eta)$ (c) Significance of $\phi$ on $\theta(\eta)$ 


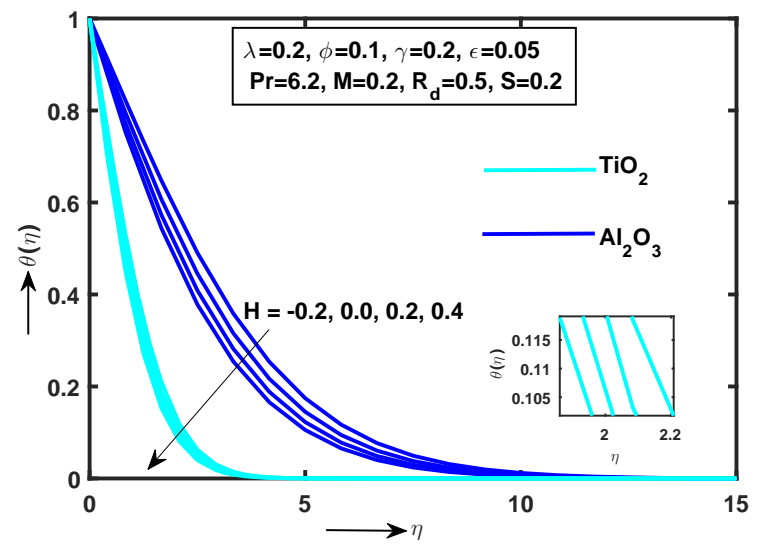

Figure 7: Significance of $H$ on $\theta(\eta)$

ascending values of $\varepsilon$ along axial and normal directions. Also, the temperature of the fluid and thermal boundary layer thickness increases with ascending values of $\varepsilon$.

Well-strategy profile $\theta(\eta)$ is illustrated in Figure 9 for distinct numerical values of $R_{d}$. It is observed that $\theta(\eta)$ increases with ascending numerical values $R_{d}$. This means that the fluid absorbs more heat from the radiation, with this both temperature and its association thermal boundary layer thickness increases.

Figures 10 displayed the effect of $\varepsilon$ against $\lambda$ on $\operatorname{Re}_{x}^{-1 / 2} N u_{x}$. It is clear that the heat transfer rate increase with ascending values of $\varepsilon$ for titanium oxide nanoparticles. This is due to the fact that, the surface heat transfer rate enhance significantly for fixed large values of $\phi$.

Figure 11 displays the influence of $S$ on heat transfer rate $\left(\operatorname{Re}_{x}^{-1 / 2} N u_{x}\right)$ against $\lambda$. It is observed that the $\operatorname{Re}_{x}^{-1 / 2} N u_{x}$ increases for increasing values of $S$. Physically, the principal of suction is associate of the heated fluid particles through stretching sheet where the buoyancy forces will slow down due to the high viscosity effect of the fluid particles.

The effect of Prandtl number on skin friction coefficient against volume fraction parameter along $y$ and $x$ axis is displayed respectively in Figures 12 (a) and (b). The local skin friction value increases with increasing values of $\operatorname{Pr}$ and $\phi$ in the presence of Copper nanoparticles.

\section{Concluding remarks}

The major outputs of the current research work are listed below

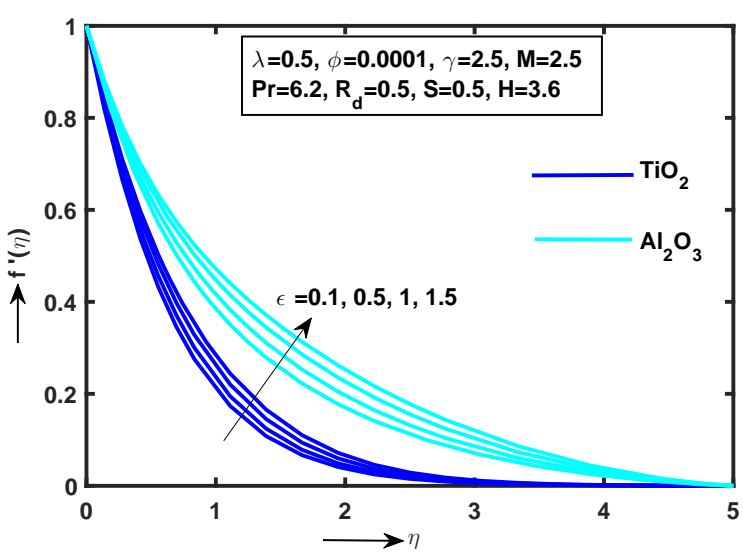

(a)

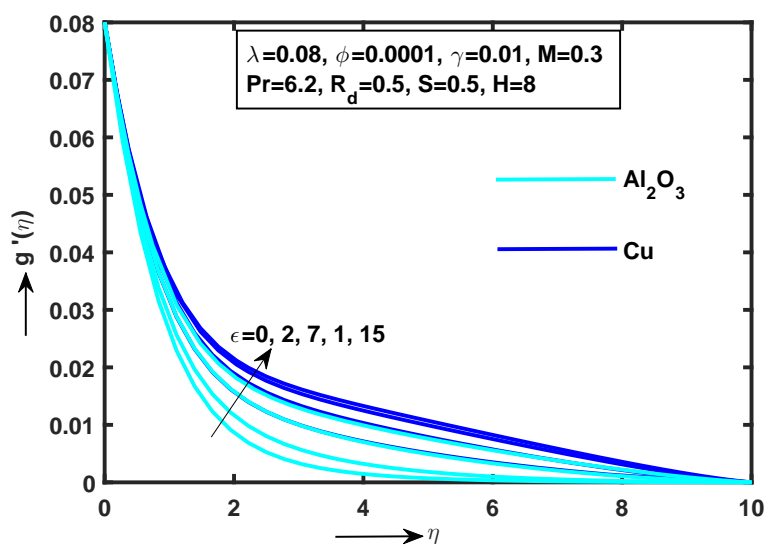

(b)

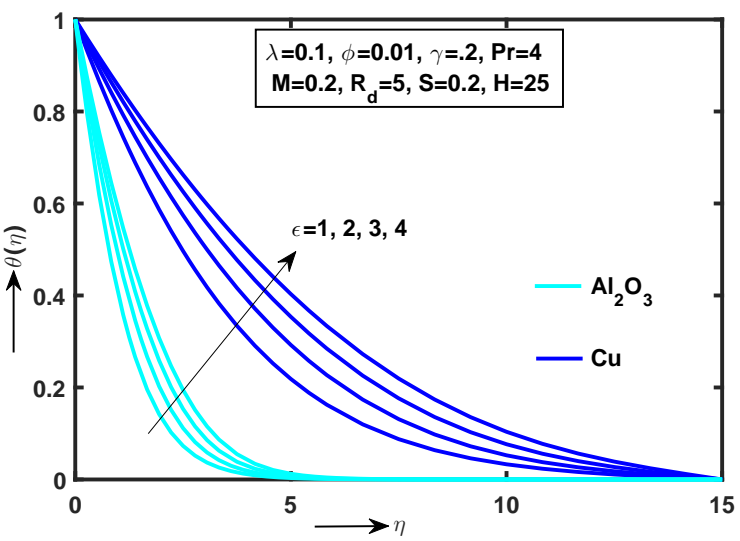

(c)

Figure 8: (a) Significance of $\epsilon$ on $f^{\prime}(\eta)$ (b) Significance of $\epsilon$ on $g^{\prime}(\eta)$ (c) Significance of $\epsilon$ on $\theta(\eta)$ 


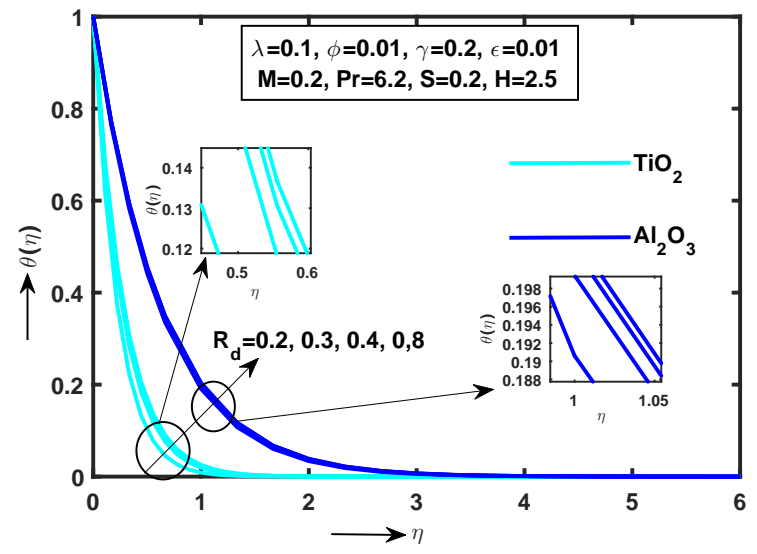

Figure 9: Significance of $R_{d}$ on $\theta(\eta)$

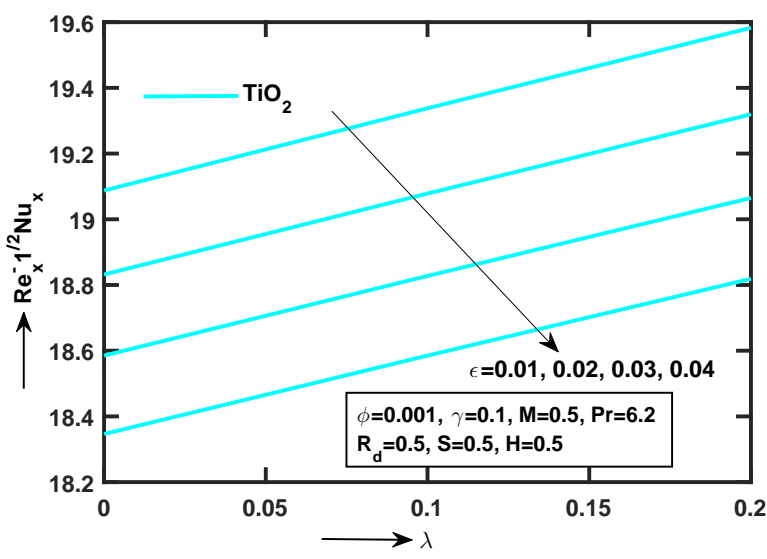

Figure 10: Significance of $\epsilon$ on $R e_{x}^{-1 / 2} N u_{x}$

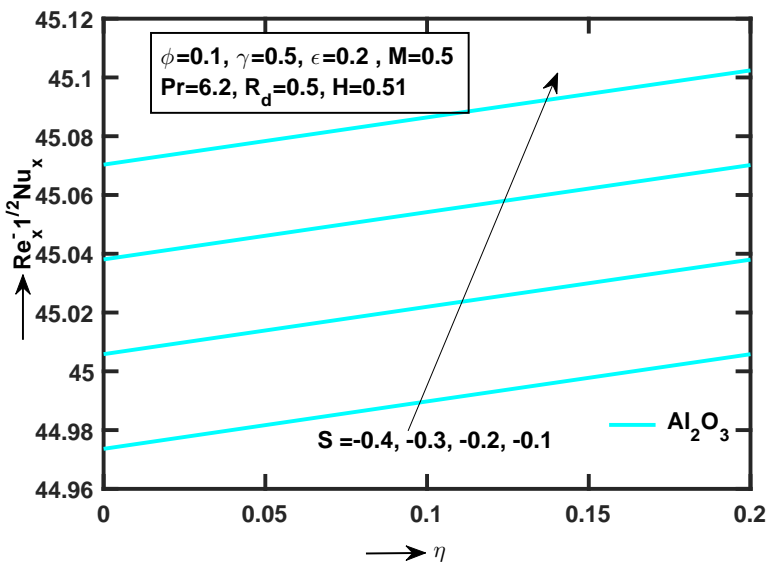

Figure 11: Significance of $S$ on $R e_{x}^{-1 / 2} N u_{x}$

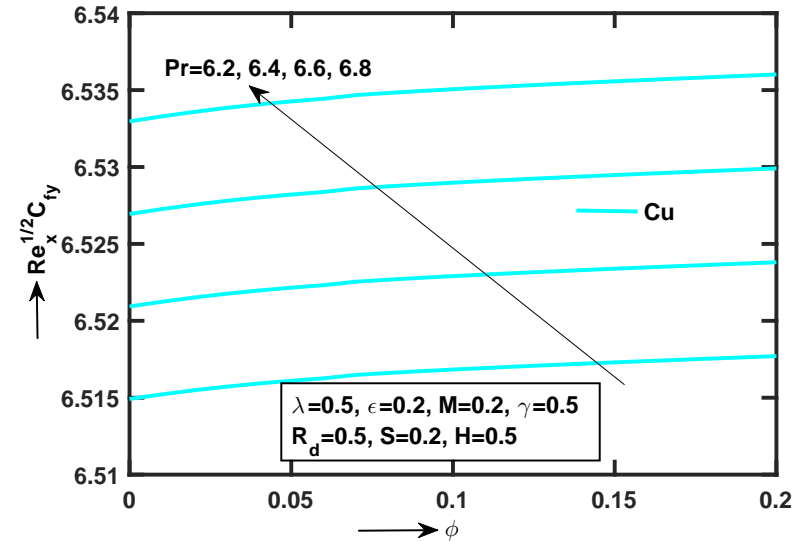

Figure 12: Significance of $\operatorname{Pr}$ on $\operatorname{Re}_{x}^{1 / 2} c_{f y}$

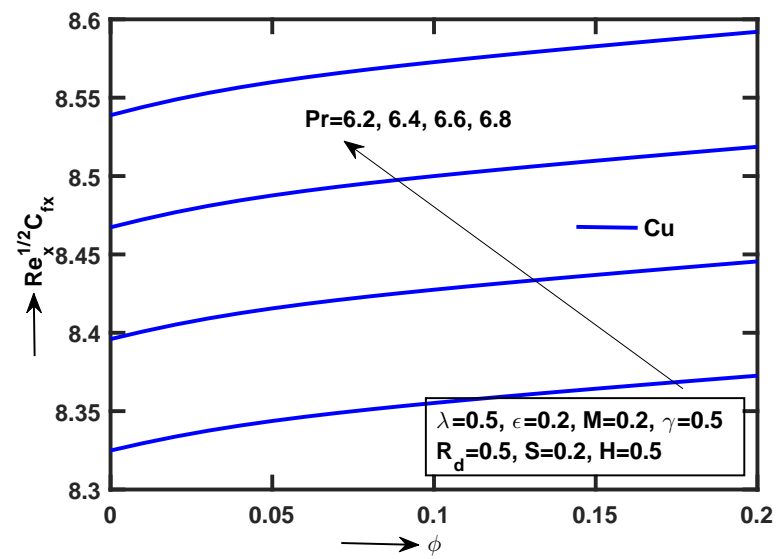

Figure 13: Significance of $\operatorname{Pr}$ on $\operatorname{Re}_{x}^{1 / 2} C_{f x}$

- The temperature and velocity of the fluid along axial direction are more in case of $\mathrm{Cu}$-water nanofluid than to that of $\mathrm{Al}_{2} \mathrm{O}_{3}$-water nanofluid.

- The heat transfer rate reduces with rising the volume fraction of nanoparticles.

- The properties of nanofluids are controlled by changing $\phi$ along $x$ and $y$-directions.

- Stretching ratio parameter shows opposite effect on axial and transverse velocities.

- The fluid temperature rises and velocity decreases with growing values of $y$ and $M$.

- Heat source parameter boost the temperature in the boundary.

- The rate of heat transfer increases for the case of injection due to high volume fraction value of nanoparticles in nanofluid. 


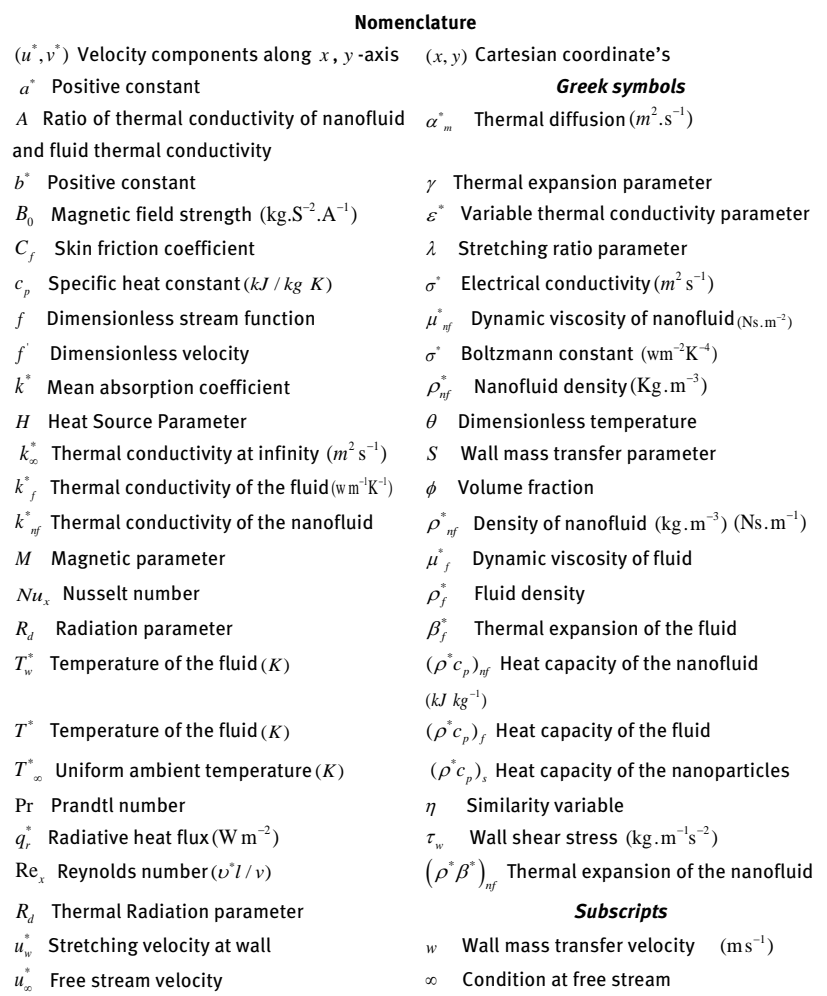

\section{References}

[1] Choi S.U., Eastman J.A., Enhancing thermal conductivity of fluids with nanoparticles, Int. Mech. Eng. Congress and Exhibition, San Francisco, CA, 1995, 231(66), 99-105.

[2] Lee S., Choi S.U.S., Li S., Eastman V., Measuring thermal conductivity of fluids containing oxide nanoparticles, J. Heat Transf., 1999, 121, 280-289.

[3] Prasad K.V., Vajravelu K., Vaidya H., Gorder R.A.V., MHD flow and heat transfer in a nanofluid over a slender elastic sheet with variable thickness, Res. Phys., 2017, 7, 1462-1474.

[4] Kasaeian A., Daneshazarian R., Mahian O., Kolsi L., Pop I., Nanofluid flow and heat transfer in porous media: a review of the latest developments, Int. J. Heat Transf., 2017, 107, 778791.

[5] Rehman A.U., Mehmood R., Nadeem S., Entropy analysis of radioactive rotating nanofluid with thermal slip, Appl. Thermal Eng., 2017, 112, 832-840.

[6] Sheikholeslami M., Sadoughi M.K., Simulation of Cuo-water nanofluid heat transfer enhancement in presence of melting surface, Int. J. Heat Mass Transf., 2018, 116, 909-919.

[7] Jafarimoghaddam A., Closed form analytic solutions to heat and mass transfer characteristics of wall jet flow of nanofluids, Thermal Sci. Eng. Progress, 2017, 4, 175-184.

[8] Bin S., Cheng P., Ruiling Z., Yang D., Hongwai L., Investigation on the flow and convective heat transfer characteristics of nanofluids in the plate heat exchanger, Exp. Thermal Fluid Sci., 2016, 76, 75-86.

[9] Ganvir R.B., Walke P.V., Kriplani V.M., Heat transfer characteristics in nanofluid - a review, Renew. Sustain. Energy Rev., 2017, 75, 451-460.
[10] Rajarathinam M., Nithyadevi N., Heat transfer enhancement of Cu-water nanofluid in an inclined porous cavity with internal heat generation, Thermal Sci. Eng. Progress, 2017, 4, 35-44.

[11] Sheikholeslami M., Rokni H.B., Effect of melting heat transfer on nanofluid flow in existence of magnetic field considering Buongiorno model, Chin. J. Phys., 2017, 55, 1115-1126.

[12] Umavathi J.C., Ojjela O., Vajravelu K., Numerical analysis of natural convective flow and heat transfer of nanofluids in a vertical rectangular duct using Darcy-Forchheimer-brinkman model, Int. J. Thermal Sci., 2017, 111, 511-524.

[13] Iqbal Z., Mehmood Z., Azhar E., Maraj E.N., Numerical investigation of nanofluids transport of Gyrotactic microorganisms submerged in water towards Riga plate, J. Mol. Liq., 2017, 234, 296-308.

[14] Khan J.A., Mustafa M., Mushtaq A., On Three-dimensional flow of nanofluids past a convectively heated deformable surface: a numerical study, Int. J. Heat Mass Transf., 2016, 94, 49-55.

[15] Khan M., Azam M., Munir A., On Unsteady Falkner-Skan flow of MHD carreau nanofluid past a static/moving wedge with convective surface condition, J. Mol. Liq., 2017, 230, 48-58.

[16] Hayat T., Muhammad T., Shehzad S.A., Alsaedi A. Three dimensional rotating flow of Maxwell nanofluid, J. Mol. Liq., 2017, 229, 495-500.

[17] Satya Narayana P.V., Lie Group Analysis for the Flow and Heat Transfer of a Nanofluid over a Stretching Sheet with Viscous Dissipation, J. Nanofluids, 2017, 6, 1181-1187.

[18] Tarakaramu N., Satya Narayana P.V., Unsteady MHD nanofluid flow over a stretching sheet with chemical reaction, IOP Conf. Series Mater. Sci. Eng., 2017, 263, 1-8.

[19] Satya Narayana P.V., Akshit S.M., Ghori J.P., Venkateswarlu B., Thermal radiation effects on an unsteady MHD nanofluid flow over a stretching sheet with non-uniform heat source/sink, J. Nanofluids, 2017, 6(5), 899-907.

[20] Meraj M.A., Shehzad S.A., Hayat T., Abbasi F.M., Alsaedi A., Darcy-Forchheimer flow of variable conductivity Jeffrey liquid with Cattaneo-Christov heat flux theory, Appl. Math. Mech., 2017, 38(4), 557-566.

[21] Hayat T., Javed M., Imtiaz M., Alsaedi A., Effect of cattaneochristov heat flux on Jeffrey fluid flow with variable thermal conductivity, Res. Phys., 2018, 8, 341-351.

[22] Ramzan M., Bilal M., Kanwal S., Chung J.D., Effects of variable thermal conductivity and non-linear thermal radiation past an Eyring-Powell nanofluid flow with chemical reaction, Comm. Theoret. Phys. 2017; 67(6).

[23] Srinivas Reddy C., Kishan N., Rashidi M.M., MHD flow and heat transfer characteristics of Williamson nanofluid over a stretching sheet with variable thickness and variable thermal conductivity, Trans. Razmadze Math. Inst., 2017, 171(2), 195211.

[24] Dyugaev A., Alteration of Thermal conductivity and viscosity of liquid by dispersing ultra-fine particles, Phys. Lett., 1985, 111A(6), 1-3.

[25] Gerdroodbary M.B., Rahimi Takami M., Ganji D.D., Investigation of thermal radiation on traditional Jeffrey-Hamel flow to stretchable convergent/divergent channels, Case Stud. Therm. Eng., 2015, 6, 28-39.

[26] Hayat T., Muhammad T., Alsaedi A., Alhuthali M.S., Magnetohydrodynamic three-dimensional flow of viscoelastic nanofluid in presence of nonlinear thermal radiation, J. Mag- 
net. Magn. Mater. 2015, 385, 222-229.

[27] Satya Narayana P.V., Harish Babu D., MHD free convective het and mass transfer past a vertical porous plate with variable temperature, Int. J. Appl. Math. Mech., 2013, 97, 66-94.

[28] Khan Md. S., Karim I., Biswas Md. H.A., Non-Newtonian MHD mixed convection power- law fluid flow over a vertical stretching sheet with thermal radiation, heat generation and chemical reaction effects, Acad. Res. Int., 2012, 3, 80-92.

[29] Venkateswarlu B., Satya Narayana P.V., Effects of thermal radiation on unsteady MHD micropolar fluid past a vertical porous plate in the presence of radiation absorption, Int. J. Eng. Sci. Comp., 2016, 6, 2259-2270.

[30] Animasaun I.L., 47nm alumina-water nanofluid flow within boundary layer formed on upper horizontal surface of paraboloid of revolution in the presence of quartic autocatalysis chemical reaction, Alexandria Eng. J., 2016, 55, 2375-2389.

[31] Boutra A., Ragui K., Labsi N., Bennacer R., Benkahla Y.K., Natural convection heat transfer of a nanofluid into a cubical enclosure: lattice Boltzmann investigation, Arab. J. Sci. Eng., 2016, 41, 1969-1980.

[32] Saghir M.Z., Ahadi A., Mohamad A., Srinivasan S., Water aluminium oxide nanofluid benchmark model, Int. J. Therm. Sci., 2016, 109, 148-158.

[33] Purusothaman A., Nithyadevi N., Oztop H.F., Divya V., Al-Salem K., Three dimensional numerical analysis of natural convection cooling with an array of discrete heaters embedded in nanofluid filled enclosure, Adv. Powder Technol., 2016, 27, 268-280.

[34] Snoussi L., Chouikh R., Ouerfelli N., Guizani A., Numerical simulation of heat transfer enhancement for natural convection

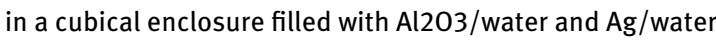
nanofluids, Phys. Chem. Liq., 2016, 54, 703-716.

[35] Sheikholeslami M., Ellahi R., Three dimensional mesoscopic simulation of magnetic field effect on natural convection of nanofluid, Int. J. Heat. Mass Transf., 2015, 89, 799-808.

[36] Kolsi L., Oztop, H.F., Alghamdi A., Abu-Hamdeh A., Borjini M.N., Ben Aissia H., A computational work on a three dimensional analysis of natural convection and entropy generation in nanofluid filled enclosures with triangular solid insert at the corners, J. Mol. Liq., 2016, 218, 260-274.
[37] Kolsi L., Hussein A.K., Borjini M.N., Mohammed H.A., Ben Aissia H., Computational analysis of three-dimensional unsteady natural convection and entropy generation in a cubical enclosure filled with water- $\mathrm{Al}_{2} \mathrm{O}_{3}$ nanofluid, Arab. J. Sci. Eng., 2014, 39, 7483-7493.

[38] Kolsi L., Kalidasan K., Alghamdi A., Borjini M.N., Kanna P.R., Natural convection and entropy generation in a cubical cavity with twin adiabatic blocks filled by aluminium oxide-water nanofluid, Numer. Heat Transf., 2016, 70, 242-259.

[39] Meng X., Li Y., Numerical study of natural convection in a horizontal cylinder filled with water-based alumina nanofluid, Nanoscale Res. Lett., 2015, 10(1), 142.

[40] Ho C.J., Liu W.K., Chang Y.S., Lin C.C., Natural convection heat transfer of alumina-water nanofluid in vertical square enclosures: an experimental study, Int. J. Therm. Sci., 2010, 49, 1345-1353.

[41] Nayak M.K., Akbar N.S., Pandey V.S., Khan Z.H., Dharmendra T., 3D free convective MHD flow of nanofluid over permeable linear stretching sheet with thermal radiation, Powder Technol., 2017, 315, 205-215.

[42] Bilal S., Rehman K., Malik M.Y., Hussain A., Khan M., Effect of temperature dependent conductivity and absorption/generation heat transfer on MHD three dimensional flow of Williamson fluid to bidirectional non-linear stretching surface, Res. Phys., 2017, 7, 204-212.

[43] Abbas W., Sayed E.A., Hall current and joule heating effects on free convection flow of a nanofluid over a vertical cone in presence of thermal radiation, Therm. Sci. 2017, 21(6A), 1-13.

[44] Brewster M.Q., Thermal radiative transfer properties, 1972, Wiley New York.

[45] Magyariand E.,, Keller B., Heat and mass transfer in the boundary layers on an exponentially stretching continuous surface, J. Phys. D Appl. Phys., 1999, 32, 577-585.

[46] Liu I.C., Wang H.H., Peng Y.F., Flow and heat transfer for threedimensional flow over an exponentially stretching surface, Chem. Eng. Comm., 2012, 200(2), 253-268. 